\title{
Some Schrödinger-type inequalities for stabilization of discrete linear systems associated with the stationary Schrödinger operator
}

\section{Zongcai Jiang ${ }^{*}$}

"Correspondence:

jiangliu81@163.com

School of Mathematics and

Information Science, Henan

University of Economics and Law,

Zhengzhou, 450046, China

\section{Springer}

\begin{abstract}
By applying some Schrödinger-type inequalities developed by Huang (Int. J. Math. 27(2):1650009, 2016), we are concerned with stabilization of discrete linear systems associated with the Schrödinger operator. Our first aim is to prove a state-dependent switching law associated with the Schrödinger operator, which is based on a convex combination. Next, we derive sufficient conditions associated with the Schrödinger operator that guarantee the uniform exponential stability of the system. Finally, we propose a necessary and sufficient condition for the stability of a system with two Schrödinger subsystems.
\end{abstract}

Keywords: Schrödinger-type inequalities; stabilization; stationary Schrödinger operator

\section{Introduction}

A switched system is composed of several subsystems and a decision rule that orchestrates switching between these subsystems. Due to their wide existence in engineering applications, this kind of dynamical systems has attracted considerable attention during the past decades. In the real word, many quantities are nonnegative [2-4]. As a special case of switched systems, a switched positive system with all subsystems associated with the Schrödinger operator being positive systems is very common in communication systems associated with the Schrödinger operator, formation flying, and rival mutation treatment system [5].

As we all know, it is necessary to address the stability and stabilization issues for switched positive systems associated with the Schrödinger operator. Generally, the synthesis problem of switched systems associated with the Schrödinger operator primarily concerns on two topics: stabilization by feedback controllers and stabilization by feedback switching law. The former topic is studied mainly based on state feedback or output feedback controller designation $[6,7]$, whereas the latter topic is proved by feedback switching law $[8$, 9]. Furthermore, there are many switched systems associated with the Schrödinger operator whose subsystems associated with the Schrödinger operator are not stable in real control systems associated with the Schrödinger operator $[1,10]$. To the best of our knowl-

(c) 2016 Jiang. This article is distributed under the terms of the Creative Commons Attribution 4.0 International License (http://creativecommons.org/licenses/by/4.0/), which permits unrestricted use, distribution, and reproduction in any medium, provided you give appropriate credit to the original author(s) and the source, provide a link to the Creative Commons license, and indicate if changes were made. 
edge, existing stabilization results on switched positive linear systems associated with the Schrödinger operator have little results referring to the state-dependent switching law, which motivates the research of this paper.

This paper investigates the boundary value problems of discrete linear systems associated with the Schrödinger operator in discrete time context, which contain unstable subsystems associated with the Schrödinger operator. The state feedback switching law is designed to guarantee the stabilization of the switched positive linear systems associated with the Schrödinger operator. The result is then applied to systems associated with the Schrödinger operator with two subsystems associated with the Schrödinger operator, and sufficient and necessary condition is also derived. The rest of paper is organized as follows. Section 2 gives introduces some preliminaries. In Section 3, we give the main results. Section 4 concludes this paper.

Throughout the paper, $\Re^{n}$ and $\Re^{n \times n}$ represent the vectors of $n$-tuples of real numbers and the space of $n \times n$ matrices with real entries, respectively, and $\mathbb{N}$ is the set of nonnegative integers. For $v$ in $\Re^{n}, v_{i}$ is the $i$ th component of $v$. The notation $v \succ 0(\succeq 0)$ means that all components of $v$ are positive (nonnegative), that is, $v_{i}>0(\geq 0)$, and $\bar{\lambda}_{v}$ and $\underline{\lambda}_{v}$ stand for the maximal and minimal components of $v$, respectively. For a matrix $A$ in $\Re^{n \times m}, a_{i j}$ is the element in the $i$ th row of the $j$ th column of $A$, and $A \succ 0(\succeq 0)$ means that all elements of $A$ are positive (nonnegative), that is, $a_{i j}>0(\geq 0)$.

\section{Preliminaries}

Consider the following discrete-time switched linear system:

$$
x(k+1)=A_{\sigma(k)} x(k), \quad k \in \mathbb{N},
$$

where $\sigma(k)$ is a piecewise constant switching signal taking values in the finite set $S=$ $\{1,2, \ldots, N\}$, and $A_{i} \in \Re^{n \times n}(i \in S)$ are system matrices.

Assumption 1 For system (2.1), $A_{i} \succeq 0$ for each $i \in S$.

Definition 1 A switching signal $\sigma$ is said to be a state-feedback switching law if it depends on system states and its past value, that is, $\sigma\left(t^{+}\right)=\sigma\left(x(t), \sigma\left(t^{-}\right)\right)$for system (2.1), where $\sigma(k+1)=\sigma(x(k), \sigma(k-1))$.

Lemma 1 ([2]) A discrete-time system

$$
x(k+1)=A x(k), \quad k \in \mathbb{N},
$$

is positive if and only if $A \succeq 0$.

Lemma 2 Let $A \succeq 0$. Then the following conditions are equivalent:

(i) $A$ is a Schur matrix;

(ii) There exists vector $v \succ 0$ in $\Re^{n}$ with $(A-I) v \prec 0$.

Proof (i) For any $i \in E, i \geq 3$,

$$
E^{i}\left\{e^{\lambda \sigma_{2}}\right\}=E^{i}\left\{e^{\lambda\left(T_{1}+\sigma_{2} \circ \theta_{T_{1}}\right)}\right\}=\frac{1}{q_{i}-\lambda} E^{1}\left\{e^{\lambda \sigma_{2}}\right\} .
$$


In the following, we compute $E^{1}\left\{e^{\lambda \sigma_{1}}\right\}$. Consider the coordinate process $W(s)$ on excursion space $(U, \mathcal{U})$. For any $i \in E$, we define

$$
\eta_{i}=\inf \{s \mid W(s)=i\}
$$

and

$$
C_{0}=\left\{W \mid W_{0} \neq 2\right\}, \quad C_{1}=\left\{W \mid W_{0}=2\right\}
$$

Obviously, $C_{0}, C_{1} \in \mathcal{U}$ and $C_{0} \cup C_{1}=U$. Let $\tau=\inf \left\{t \mid \beta_{t}>\sigma_{2}\right\}$ for any $t>0$, and

$$
Z_{t}^{1}=\sharp\left\{s \mid s \in D_{p}, s \leq t, Y_{s} \in C_{1}\right\} .
$$

Then we have

$$
\begin{aligned}
P^{1}\{\tau>t\} & =\hat{P}\left\{Z_{t}^{1}=0\right\} \\
& =e^{-\hat{P}\left(W_{0}=2\right) t}=e^{-t} .
\end{aligned}
$$

So we know that $\tau$ is an exponential random variable with mean 1 . Hence, we have

$$
\begin{aligned}
E^{1}\left\{e^{\lambda \sigma_{2}}\right\} & =E^{1}\left[\exp \left\{\lambda\left(\sum_{s \in G}\left(I_{\left(0, \sigma_{2}\right]}(s) \sigma_{\infty} I_{\left\{C_{0}\right\}}\left(Y_{s}\right)\right) \circ \theta_{s}\right)\right\}\right] \\
& =E^{1}\left\{\exp \left[\lambda \beta_{\tau}\right]\right\} \\
& =\int_{0}^{\infty} E^{1}\left\{e^{\lambda \beta_{t}}\right\} e^{-t} d t .
\end{aligned}
$$

From the computation of a Poisson point process we know that

$$
\begin{aligned}
E^{1}\left\{e^{\lambda \beta_{t}}\right\} & =E^{1}\left\{e^{\lambda z_{t}^{1}}\right\} \\
& =\exp \left\{t \hat{P}\left(\left(e^{\lambda \sigma_{1}}-1\right) I_{\left\{C_{1}\right\}}\left(Y_{s}\right)\right)\right\},
\end{aligned}
$$

which yields that

$$
\hat{P}\left(\left(e^{\lambda \sigma_{1}}-1\right) I_{\left\{C_{1}\right\}}\left(Y_{s}\right)\right)=\sum_{k=3}^{\infty} E^{k}\left[e^{\lambda \sigma_{1}}-1\right]=\sum_{k=3}^{\infty} \frac{\lambda}{q_{k}-\lambda} .
$$

Hence, we have

$$
E^{1}\left\{e^{\lambda \sigma_{1}}\right\}=\int_{0}^{\infty} \exp t\left\{\sum_{k=3}^{\infty} \frac{\lambda}{q_{k}-\lambda}-1\right\} d t=\frac{1}{1-\sum_{k=3}^{\infty} \frac{\lambda}{q_{k}-\lambda}} .
$$

Therefore, if $0<\lambda<\frac{1}{\sum_{n=3}^{\infty}\left(q_{n}-q_{2}\right)^{-1}} \wedge q_{2}$, then we have $E^{1}\left[e^{\lambda \sigma_{2}}\right]<\infty$. So $E^{i}\left\{e^{\lambda \sigma_{1}}\right\}<\infty$. It is easy to see that $p_{i j}(t)$ is exponentially ergodic.

(ii) By Lemma 1 and the previous results we have that if $i \geq 3$, then $\tau_{2}^{+}=\sigma_{2}$. Let $\lambda$ satisfy $0<\lambda<\frac{1}{\sum_{n=3}^{\infty}\left(q_{n}-q_{1}\right)^{-1}} \wedge q_{2}$. Then, for any $i \in E$, we have

$$
E^{i}\left\{e^{\lambda \tau_{1}^{+}}\right\}=E^{i}\left\{e^{\lambda \sigma_{2}}\right\}<\infty .
$$


Put $m=2$ in Theorem 1. By using a computable method of (2.1) we know that

$$
\begin{aligned}
E^{2}\left\{e^{\lambda \tau_{1}^{+}}\right\} & =E^{2}\left\{e^{\lambda\left(T_{1}+\sigma_{2} \circ T_{1}\right)}\right\} \\
& =E^{2}\left[e^{\lambda T_{1}}\right] E^{1}\left[e^{\lambda \sigma_{2}}\right] \\
& =\frac{1}{q_{1}-\lambda} \frac{1}{1-\sum_{k=3}^{\infty} \frac{\lambda}{q_{k}-\lambda}} .
\end{aligned}
$$

So Lemma 2 is proved.

For system (2.2), if there exists a vector $v \succ 0$ with $v \in \mathfrak{R}^{n}$ such that $\left(A-I_{n}\right) v \prec 0$, then $V=v^{T} x$ is said to be a linear copositive Lyapunov function of system (2.2).

Next, we give an example to introduce our results.

Example 1 Consider system (2.1) with two subsystems associated with the Schrödinger operator described by

$$
A_{1}=\left(\begin{array}{ll}
0.5 & 1.4 \\
1.5 & 1.2
\end{array}\right), \quad A_{2}=\left(\begin{array}{ll}
1.3 & 1.5 \\
1.4 & 0.6
\end{array}\right)
$$

For the first subsystem matrix $A_{1}$, there does not exist a vector $v_{1} \succ 0$ such that $\left(A_{1}-\right.$ $I) v_{1} \prec 0$. Similarly, there does not exist a vector $v_{2} \succ 0$ such that $\left(A_{2}-I\right) v_{2} \prec 0$.

It is easy to verify that these two subsystem matrices are not Schur matrices. Despite this disadvantage, we find that there exist some combinations $A_{0}$ of $A_{1}$ and $A_{2}$ that are positive and Schur matrices, that is, $A_{0}=\lambda_{1} A_{1}+\lambda_{2} A_{2}$ is a positive Schur matrix, where $\lambda_{1}, \lambda_{2} \in(0,1)$ and $\lambda_{1}+\lambda_{2}=1$.

For example, choose $\lambda_{1}=0.5$ and $\lambda_{2}=0.5$. We obtain

$$
A_{0}=\left(\begin{array}{cc}
0.9 & 1.45 \\
1.45 & 0.9
\end{array}\right)
$$

Note that there exists a vector $v \succ 0$ in $\Re^{n}$ with $\left(A_{0}-I\right) v \prec 0$. Thus, $A_{0}$ is a positive and stable matrix.

This example contains an instantaneous state.

Suppose that $q_{2}, q_{3}, \ldots$ are sequences of positive real numbers and consider the following Q-matrix:

$$
Q=\left(\begin{array}{ccccc}
-\infty & 1 & 1 & 1 & \ldots \\
q_{2} & -q_{2} & 0 & 0 & \ldots \\
q_{3} & 0 & -q_{3} & 0 & \ldots \\
q_{4} & 0 & 0 & -q_{4} & \ldots \\
\vdots & \vdots & \vdots & \vdots & \ldots
\end{array}\right)
$$

where $\sum_{i=2}^{\infty} q_{i}{ }^{-1}<\infty$. This matrix is called the Kolmogorov matrix. There exist infinitely many dishonest processes with this $Q$-matrix. In $[9,10]$, it is shown that the process with following resolvent is the only honest one:

$$
R_{11}(\lambda)=\frac{1}{\lambda}\left(1+\sum_{k=2}^{\infty} \frac{1}{\lambda+q_{k}}\right)^{-1}
$$




$$
\begin{aligned}
& R_{1 j}(\lambda)=R_{11}(\lambda) \cdot \frac{1}{\lambda+q_{j}}, \quad j \geq 2, \\
& R_{i 1}(\lambda)=\frac{q_{i}}{\lambda+q_{i}} \cdot R_{11}(\lambda), \quad i \geq 2, \\
& R_{i j}(\lambda)=\frac{q_{i}}{\lambda+q_{i}} \cdot R_{11}(\lambda) \cdot \frac{1}{\lambda+q_{j}}+\frac{\delta_{i j}}{\lambda+q_{j}}, \quad i, j \geq 2,
\end{aligned}
$$

where $\lambda>0$. Let the state space be $E=\{1,2,3, \ldots\}$. Obviously, the transition function $p_{i j}(t)$ that corresponds to the resolvent is the only honest one. Though this chain is weakly symmetric, so far its convergence rate is still unknown because of its instantaneous state.

Next, we discusses the stability for system (2.1). Define the following stable convex combination of the system matrices:

$$
\bar{A}_{0}=\sum_{i \in S} \bar{w}_{i} A_{i}
$$

where

$$
\sum_{i \in S} \bar{w}_{i}=1, \quad \bar{w}_{i} \in(0,1)
$$

Assumption 2 There exists a vector $\bar{v} \in \mathfrak{R}^{n}$ with $\bar{v} \succ 0$ such that

$$
\left(\bar{A}_{0}-I_{n}\right)^{T} \bar{v} \prec 0 .
$$

Without loss of generality, let

$$
\left(\bar{A}_{0}-I_{n}\right)^{T} \bar{v}=-\overline{\mathbf{e}},
$$

where $\overline{\mathbf{e}} \in \Re^{n}$ with $\overline{\mathbf{e}} \succ 0$, and $\frac{\lambda_{\overline{\mathbf{e}}}}{\bar{\lambda}_{\bar{v}}} \leq 1$.

Denote

$$
\bar{\ell}_{i}=\left(A_{i}-I_{n}\right)^{T} \bar{v}, \quad i \in S .
$$

Combing (2.3), (2.4), (2.5), (2.6), and (2.7), we get

$$
\left[\left(\bar{w}_{1} A_{1}+\cdots+\bar{w}_{N} A_{N}\right)-\left(\bar{w}_{1}+\cdots+\bar{w}_{N}\right) I_{n}\right]^{T} \bar{v}=\bar{w}_{1} \bar{\ell}_{1}+\cdots+\bar{w}_{N} \bar{\ell}_{N}=-\overline{\mathbf{e}}
$$

\section{Switching rule 1}

(i) For any initial state $x\left(k_{0}\right)=x_{0}$, we select

$$
\sigma\left(k_{0}\right)=\underset{i \in S}{\arg \min }\left\{x_{0}^{T} \bar{\ell}_{i}\right\}
$$

(ii) The first switching time instant is selected as

$$
k_{1}=\inf \left\{k \geq k_{0} \mid x(k)^{T} \bar{\ell}_{\sigma\left(k_{0}\right)}>-r_{\sigma\left(k_{0}\right)} x(k)^{T} \overline{\mathbf{e}}\right\} .
$$


Thus, the switching index can be determined by

$$
\sigma\left(k_{1}\right)=\underset{i \in S}{\arg \min }\left\{x\left(k_{1}\right)^{T} \bar{\ell}_{i}\right\}
$$

(iii) The switching index sequences are defined by

$$
\begin{aligned}
& \qquad k_{j+1}=\inf \left\{k \geq k_{j} \mid x(k)^{T} \bar{\ell}_{\sigma\left(k_{j}\right)}>-r_{\sigma\left(k_{j}\right)} x(k)^{T} \overline{\mathbf{e}}\right\}, \\
& \sigma\left(k_{j+1}\right)=\underset{i \in S}{\arg \min }\left\{x\left(k_{j+1}\right)^{T} \bar{\ell}_{i}\right\} \\
& \text { where } r_{\sigma\left(k_{j}\right)} \in(0,1) \text { and } j \in \mathbb{N} \text {. }
\end{aligned}
$$

\section{Main results}

Theorem 1 Under Assumption 2 and Switching rule 1, system (2.1) is uniformly exponentially stable.

Proof Choose $V(k, x(k))=x(k)^{T} \bar{v}$. For any $k \in\left[k_{m}, k_{m+1}\right)$, by system (2.1) it follows that

$$
x(k)^{T} \bar{v}=x(k-1)^{T} A_{\sigma\left(k_{m}\right)}^{T} \bar{v},
$$

which, together with (2.1) and (3.1), gives that

$$
x(k)^{T} \bar{v}=x(k-1)^{T} \bar{\ell}_{\sigma\left(k_{m}\right)}+x(k-1)^{T} \bar{v} .
$$

By (3.2) and (iii) of Switching rule 1 we know that

$$
x(k)^{T} \bar{v} \leq-r_{\sigma\left(k_{m}\right)} x(k-1)^{T} \overline{\mathbf{e}}+x(k-1)^{T} \bar{v}
$$

Using (3.3) and (iii) of Switching rule 1, we can obtain the following inequality

$$
V(k, x(k))-V(k-1, x(k-1)) \leq-\frac{r_{\sigma\left(k_{m}\right)} \underline{\lambda} \overline{\mathbf{e}}}{\bar{\lambda}_{\bar{v}}} V(k-1, x(k-1))
$$

So (3.3) and (3.4) give that

$$
V(k, x(k)) \leq\left(1-\frac{r_{\sigma\left(k_{m}\right)} \underline{\lambda}_{\overline{\mathbf{e}}}}{\bar{\lambda}_{\bar{v}}}\right) V(k-1, x(k-1))
$$

Since $\frac{\lambda}{\bar{\lambda}_{\bar{v}}} \leq 1$ and $r_{\sigma\left(k_{m}\right)} \in(0,1)$, we have that

$$
1-\frac{r_{\sigma\left(k_{m}\right)} \underline{\lambda}_{\overline{\mathbf{e}}}}{\bar{\lambda}_{\bar{v}}} \in(0,1)
$$

With (3.5) in mind, using (3.6), we get that

$$
V(k, x(k)) \leq\left(1-\frac{r_{\sigma\left(k_{m}\right)} \underline{\lambda}_{\overline{\mathbf{e}}}}{\bar{\lambda}_{\bar{v}}}\right)^{k-k_{m}} V\left(k_{m}, x\left(k_{m}\right)\right)
$$


Repeating the above steps in (3.4)-(3.7), it straightforwardly follows that

$$
V(k, x(k)) \leq\left(1-\frac{r_{\sigma\left(k_{m}\right)} \underline{\lambda}_{\overline{\mathbf{e}}}}{\bar{\lambda}_{\bar{v}}}\right)^{k-k_{m}}\left(1-\frac{r_{\sigma\left(k_{m-1}\right)} \underline{\lambda}_{\overline{\mathbf{e}}}}{\bar{\lambda}_{\bar{v}}}\right)^{k_{m}-k_{m-1}}
$$

From (3.8) and (3.7) it follows that

$$
1-\frac{r_{\sigma\left(k_{i}\right)} \underline{\lambda}_{\overline{\mathbf{e}}}}{\bar{\lambda}_{\bar{v}}} \in(0,1), \quad i=0,1, \ldots, m
$$

Choose $\eta=\max _{i=0,1, \ldots, m}\left\{1-\frac{r_{\sigma\left(k_{i}\right)} \bar{\lambda}_{\bar{e}}}{\bar{\lambda}_{\bar{v}}}\right\}$. So

$$
V(k, x(k)) \leq \eta^{k-k_{0}} V\left(k_{0}, x\left(k_{0}\right)\right)
$$

which immediately gives that

$$
x(k)^{T} \bar{v} \leq \eta^{k-k_{0}} x\left(k_{0}\right)^{T} \bar{v} .
$$

So

$$
\|x(k)\| \leq \alpha \eta^{k-k_{0}}\left\|x\left(k_{0}\right)\right\|
$$

where $\alpha=\frac{\bar{\lambda}_{\bar{v}}}{\underline{\lambda}_{\bar{v}}}$.

Thus, system (2.1) is uniformly exponentially stable.

Remark 1 By the properties of discrete-time systems associated with the Schrödinger operator and (ii) and (iii) of Switching rule 1, it is not hard to find that the dwell time in each time interval is at least greater than 1 . So there exist finite switchings in any finite time interval. Thus, Switching rule 1 is well defined.

Corollary 1 Suppose $N=2$. Consider the stability of system (2.1) in the sense of Lyapunov function. Then system (2.1) is stable if and only if Assumption 2 holds.

Proof Here we only give the proof of "only if". The stability of system (2.1) implies that there exists an LCLF $V(k, x(k))=x^{T}(k) \bar{v}$ such that

$$
V(k+1, x(k+1))-V(k, x(k))<-\bar{\zeta} x^{T}(k) \overline{\mathbf{e}^{\prime}}
$$

or

$$
V(k+1, x(k+1))-V(k, x(k))<-\bar{\varsigma} x^{T}(k) \overline{\mathbf{e}^{\prime}},
$$

where $\bar{v} \succ 0, \overline{\mathbf{e}^{\prime}} \succ 0$, and $\bar{\zeta}$ is a positive real number.

That is to say, $x^{T}(k)\left(A_{1}^{T}-I_{2}\right) \bar{v}<-\bar{\zeta} x^{T}(k) \overline{\mathbf{e}^{\prime}}$ whenever $x^{T}(k)\left(A_{2}^{T}-I_{2}\right) \bar{v} \geq-\bar{\zeta} x^{T}(k) \overline{\mathbf{e}^{\prime}}$, and $x^{T}(k)\left(A_{2}^{T}-I_{2}\right) \bar{v}<-\bar{\zeta} x^{T}(k) \overline{\mathbf{e}^{\prime}}$ whenever $x^{T}(k)\left(A_{1}^{T}-I_{2}\right) \bar{v} \geq-\bar{\zeta} x^{T}(k) \overline{\mathbf{e}^{\prime}}$. We only prove the first case since the second case can be derived as the first one. By the compactness theorem there exists a positive real number $\bar{\mu}$ such that $-x^{T}(k)\left(A_{1}^{T}-I_{2}\right) \bar{v}-\bar{\zeta} x^{T}(k) \overline{\mathbf{e}^{\prime}}>\bar{\mu}$. Between 
any two consecutive switching instants, $x(k)$ is bound. Thus, there exists a positive real number $\bar{\kappa}$ such that

$$
\bar{\kappa} \geq x^{T}(k)\left(A_{2}^{T}-I_{2}\right) \bar{v}+\bar{\zeta} x^{T}(k) \overline{\mathbf{e}^{\prime}}>0 .
$$

Choose $\bar{\varepsilon}=\frac{\bar{\mu}}{\bar{\kappa}}$. We obtain that $-x^{T}(k)\left(A_{1}^{T}-I_{2}\right) \bar{v}-\bar{\zeta} x^{T}(k) \overline{\mathbf{e}^{\prime}}-\bar{\varepsilon}\left(x^{T}(k)\left(A_{2}^{T}-I_{2}\right) \bar{v}+\right.$ $\left.\bar{\zeta} x^{T}(k) \overline{\mathbf{e}^{\prime}}\right)>-x^{T} A_{1}^{T} v-\bar{\zeta} x^{T} \mathbf{e}^{\prime}-\bar{\mu}>0$, which yields that

$$
x^{T}\left[\left(A_{1}^{T}-I_{2}\right)+\bar{\varepsilon}\left(A_{2}^{T}-I_{2}\right)\right] \bar{v}<-(1+\bar{\varepsilon}) \bar{\zeta} x^{T} \overline{\mathbf{e}^{\prime}} .
$$

Define $\bar{w}_{1}=\frac{1}{1+\bar{\varepsilon}}$ and $\bar{w}_{2}=\frac{\bar{\varepsilon}}{1+\bar{\varepsilon}}$. We have $x^{T}\left[A_{0}-I_{2}\right] \bar{v}<-\bar{\zeta} x^{T} \overline{\mathbf{e}}^{\prime}$, where $A_{0}=\bar{w}_{1} A_{1}^{T}+\bar{w}_{2} A_{2}^{T}$. This inequality implies that Assumption 2 holds.

\section{Conclusions}

In this paper, we consider the stabilization problem of discrete-time switched positive linear systems associated with the Schrödinger operator that contain unstable subsystems associated with the Schrödinger operator. We present a state-dependent switching law guaranteeing that the systems associated with the Schrödinger operator are uniformly exponentially stable. Furthermore, we establish a necessary and sufficient condition for the stabilization of the system with two subsystems associated with the Schrödinger operator.

\section{Competing interests}

The author declares that she has no competing interests.

\section{Acknowledgements}

This work was supported by the Science and Technology Research Project of Henan Province (No. 152102310089)), the Key Scientific Research Projects for Colleges and Universities of Henan Province (No. 17A120006) and the Humanities and Social Sciences Research Project of Henan Provincial Department of Education (No. 2017-ZZJH-014).

Received: 25 July 2016 Accepted: 19 September 2016 Published online: 07 October 2016

\section{References}

1. Huang, J: Growth property at infinity of the maximum modulus with respect to the Schrödinger operator. Int. J. Math. 27(2), 1650009 (2016)

2. Berman, A, Neumann, M, Stern, R: Nonnegative Matrices in Dynamics Systems. Wiley, New York (1989)

3. Luenberger, D: Introduction to Dynamic Systems Associated with the Schrödinger Operator: Theory, Models, and Applications. Wiley, New York (1979)

4. Farina, L, Rinaldi, S: Positive Linear Systems Associated with the Schrödinger Operator: Theory and Applications. Wiley, New York (2000)

5. Jadbabaie, A, Lin, J, Morse, A: Co-ordination of groups of mobile autonomous agents using nearest neighbor rules. IEEE Trans. Autom. Control 48(6), 988-1001 (2003)

6. Yan, Z: Sufficient conditions for non-stability of stochastic differential systems. J. Inequal. Appl. 2015, 377 (2015)

7. Wang, J, Pu, J, Huang, B, Shi, G: Boundary value behaviors for solutions of the equilibrium equations with angular velocity. Bound. Value Probl. 2015, 230 (2015)

8. Yang, D, Ren, Y: Dirichlet problem on the upper half space. Proc. Indian Acad. Sci. Math. Sci. 124(2), 175-178 (2014)

9. Rami, M, Tadeo, F, Benzaouia, A: Control of constrained positive discrete systems associated with the Schrödinger operator. In: Proceedings of the 2007 American Control Conference, New York, USA, pp. 5851-5856 (2007)

10. Yan, Z, Yan, G, Miyamoto, I: Fixed point theorems and explicit estimates for convergence rates of continuous time Markov chains. Fixed Point Theory Appl. 2015, 197 (2015) 